\title{
Clinicopathological correlation of primary and recurrent choroidal neovascularisation following surgical excision in age related macular degeneration
}

\author{
Alessandro A Castellarin, Ma'an A Nasir, Ilene K Sugino, Marco A Zarbin
}

\begin{abstract}
Aims/background-Fluorescein angiography and histopathological findings were correlated in two patients with recurrent choroidal neovascular membranes (CNVs) in an attempt to gain insight into the possible causes of recurrent CNVs and into the healing response after CNV excision.

Methods-Two patients with recurrent CNVs underwent repeat excision, and the excised tissue was studied with light and electron microscopy.

Results-Incomplete CNV excision probably led to the recurrences. The portion initially excised appears to have been anterior to the RPE in case 1 . In both cases, recurrent CNVs contained RPElike cells suggesting that native RPE can repopulate the dissection bed. The tissue excised at the second operation contained areas with hyperplastic RPE and fragments of Bruch's membrane (external to the RPE basement membrane) in a matrix of fibrillar collagen and fibrocytes, suggesting that initial removal of the CNV can be followed by an abnormal anatomical arrangement of RPE and scarring of Bruch's membrane.
\end{abstract}

Conclusions-Abnormal resurfacing of the dissection bed by RPE and fibroblasts may underlie, in part, the limited visual outcome often seen after surgical excision of CNVs in age related macular degeneration.

(Br f Ophthalmol 1998;82:480-487)

USA

A A Castellarin

I K Sugino

M A Zarbin

Department of Ophthalmology, University of

California, San

Francisco, San

Francisco, CA, USA

M A Nasir

Correspondence to: Marco A Zarbin, MD, $\mathrm{PhD}$, University of Medicine and Dentistry, New Jersey

Medical School, Department of Ophthalmology, 90 Bergen Street, DOC 6th floor, Newark, NJ 07103-2499, USA.

Accepted for publication 27 November 1997 AMD such as interferon alfa or thalidomide therapy, low dose radiation therapy, and surgi- cal excision of CNVs. ${ }^{7-12}$ Visual recovery following surgery for subfoveal CNVs in patients with AMD is generally limited, but visual acuity is often stabilised. ${ }^{81-15}$ A multicentre, prospective, randomised study will compare surgery with laser therapy and with observation for subfoveal CNVs in AMD. ${ }^{13}$

The published reports from uncontrolled studies indicate that persistence and/or recurrence of subfoveal CNVs also occurs after subretinal CNV excision. Berger and Kaplan noted CNV persistence (as a result of attempting to preserve subfoveal retinal pigment epithelium) in three of $19(16 \%)$ eyes with AMD with average follow up of 3.7 months. ${ }^{8}$ Lambert and associates did not note persistent or recurrent CNVs in their first 10 cases during the first 6 months of follow up. ${ }^{11}$ Thomas and coworkers reported a $27 \%$ recurrence rate among a group of 41 patients with AMD with average follow up of 15 months. ${ }^{12}$ Among 20 eyes followed for 12 months or more, the recurrence rate was $46 \%$. Ormerod et al reported a 2 year recurrence rate of $40 \%$ among 10 patients (10 eyes) with AMD. ${ }^{13}$ Melberg and Thomas ${ }^{16}$ found a $44 \%$ recurrence rate among a group of 117 patients with presumed ocular histoplasmosis syndrome who underwent CNV excision.

We report a clinicopathological correlation in two patients with AMD who underwent primary and secondary CNV excision. One patient was noted to have persistent CNV 7 weeks after surgery, and another developed recurrent CNV 7 months after surgery. These two patients underwent repeat $\mathrm{CNV}$ excision. The excised tissue was studied histologically, comparing the primary and secondary CNV specimens. Fluorescein angiographic and pathological findings were correlated in order to obtain insight into the possible causes of CNV recurrence and to study the subretinal healing response that occurs after subfoveal CNV excision. A preliminary report of these results has been presented previously. ${ }^{17}$

\section{Methods}

CNV size was estimated using the preoperative fluorescein angiogram and assuming a horizontal optic nerve head diameter of $1500 \mu \mathrm{m}$. Impaired choriocapillaris perfusion was judged to be present if the choriocapillaris filling was delayed in the early venous filling phase of the angiogram, and non-perfusion was judged to be present if the filling defect persisted in the late venous filling phase of the angiogram. 
SURGICAL TECHNIQUE

Both patients were operated on by the same surgeon (MAZ). A conventional three port vitrectomy was done. The posterior hyaloid was excised at the time of the primary surgery. The projected preoperative fluorescein angiogram was used to direct the dissection in an attempt to insure complete removal of the $\mathrm{CNV}$ at the first operation. Using a 33 gauge infusion cannula, a retinotomy was made near the neovascular complex, and a small amount (less than $0.5 \mathrm{ml}$ ) of balanced salt solution was injected into the subretinal space to protect the neurosensory retina during the dissection and removal of the CNV. Adhesions were not encountered in these cases. The 33 gauge infusion cannula was used to create a cleavage plane between the $\mathrm{CNV}$ and the underlying
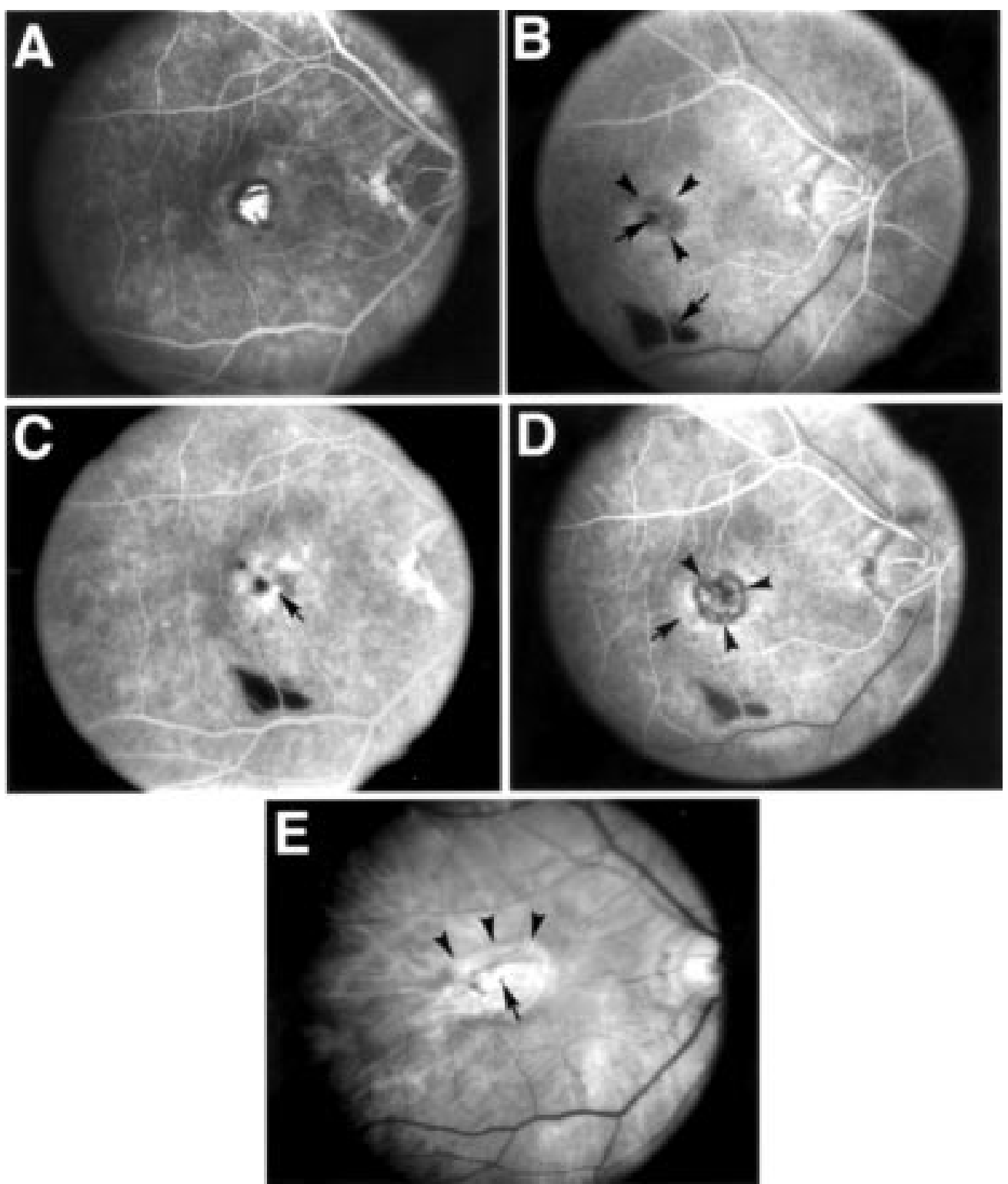

Figure 1 Case 1. (A) Preoperative fluorescein angiogram showing well demarcated subfoveal $C N V$ with border of elevated blocked fluorescence. (B) Fluorescein angiogram taken 3 weeks after CNV excision demonstrates absence of the well demarcated CNV (arrowheads) and blockage due to subretinal blood (arrows). (C) Late frame of the angiogram in (B) reveals punctate hyperfluorescence and leakage in location of the excised CNV (arrow). (D) Fluorescein angiogram taken 7 weeks after surgery reveals a persistent subfoveal CNV (arrowheads) with a faint wreath of hyperfluorescence (arrow). (E) Fundus photograph taken 3 months after the second operation showing RPE-choriocapillaris atrophy in the centre of the dissection bed (arrow) and persistent choroidal neovascularisation at the perimeter of the dissection (arrowheads) with associated subretinal haemorrhage. tissue. The edge of the CNV was grasped and elevated with a 25 gauge forceps. With the edge CNV grasped, gentle side to side traction was applied, and the CNV separated gradually from the surrounding retinal pigment epitheexcision was performed while the intraocular pressure was elevated sufficiently to produce central retinal artery pulsation for no longer than 10 minutes. The neovascular complex was removed as a single piece of tissue in all four procedures. As judged by the preoperative excised tissue bore a strong resemblance to the entire CNV (classic and occult components). The vitreous cavity was washed out for 5 minutes to remove any liberated RPE. A fluid-air
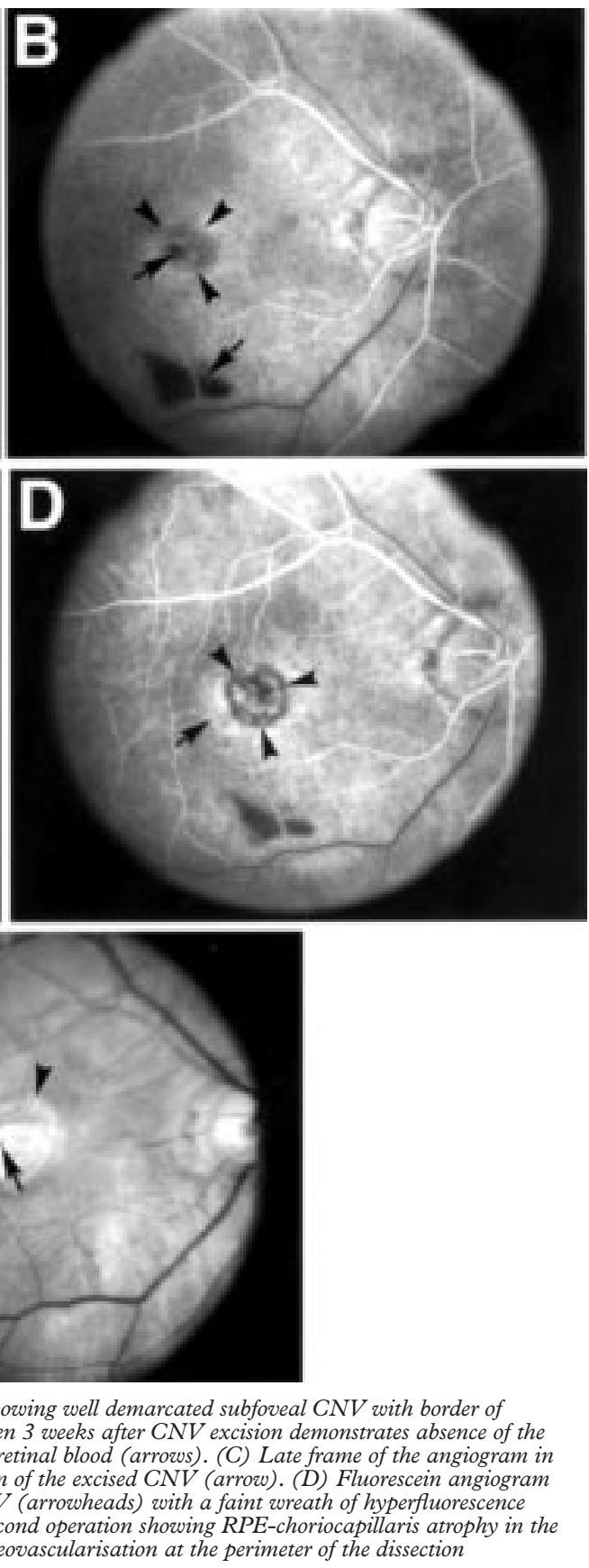

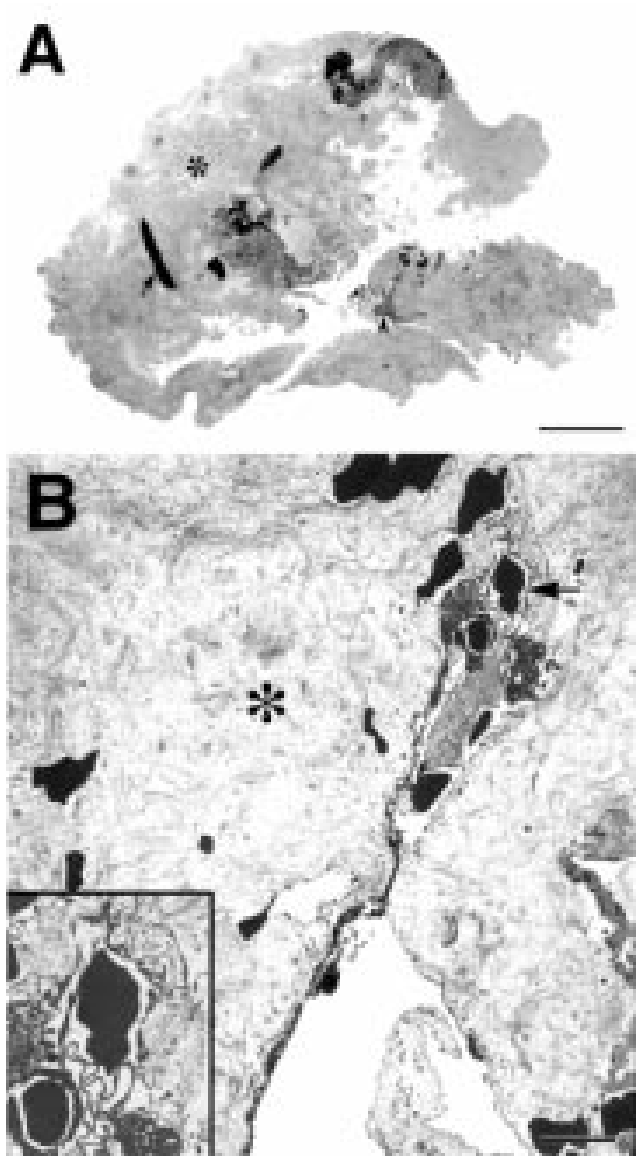

Figure 2 Case 1. (A) Photomicrograph of excised primary CNV shows loose (asterisk) and packed (arrow) collagen. Bar $=100 \mu \mathrm{m}$. Arrowhead shows location of $(B)$.

(B) Transmission electron micrograph of $(A)$ reveals loosely organised collagen (asterisk) and blood filled,

non-endothelial lined vascular channels (arrow). High magnification inset $(2 \times$ magnification) shows vascular channels. Bar $=5 \mu \mathrm{m}$.

completely. We did not treat the retinotomy with laser in either surgery in case 1 . We lasered around the retinotomy after the excision of both the primary and recurrent CNV in case 2 . In three of the four procedures, we used air to tamponade the retinotomies. At the end of the recurrent $\mathrm{CNV}$ excision in case 1 , a one clock hour superotemporal dialysis was noted and was treated with cryotherapy and an encircling No 240 band. This eye was filled with $20 \% \mathrm{SF}_{6}$. The patients maintained a strict face down posture postoperatively until the air-gas bubble resorbed.

CNV HISTOLOGY AND ULTRASTRUCTURE

For microscopic analysis, the specimens were fixed in formol saline followed by half strength Karnovsky's fixative. Routine post fixation and embedding were as follows. Samples were rinsed with several changes of phosphate buffered sucrose and post fixed in $2 \%$ osmium tetroxide. This was followed by rinsing in the same buffered sucrose, dehydration in ethanol, and infiltration into Epon. Tissue was polymerised for 2 days in a $60^{\circ} \mathrm{C}$ oven. For light microscopy, semithin sections were stained with toluidine blue. For transmission electron microscopy, specimens were thin sectioned on a RMC MT-7 ultramicrotome, and sections
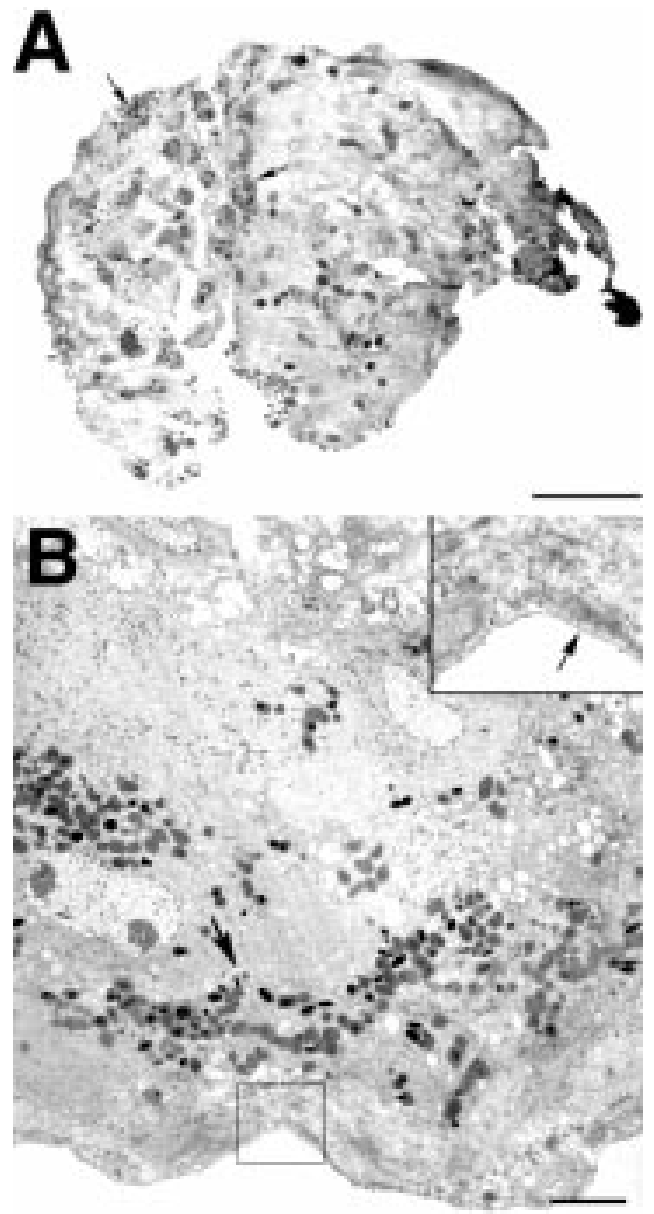

Figure 3 Case 1. (A) Photomicrograph of excised recurrent $C N V$ shows numerous presumed RPE cells (arrows) in a collagenous matrix. Bar $=50 \mu \mathrm{m}$. (B) Transmission electron micrograph of $(A)$ reveals the external surface of the CNV lined by presumed RPE (arrow). Bar $=5 \mu \mathrm{m}$. Inset $(2 \times$ magnification) shows thin (possibly newly formed) basement membrane (arrow). Bar $=4 \mu \mathrm{m}$.

were placed onto large slot grids. Grids were stained with uranyl acetate and lead citrate. Samples were examined and photographed on a Zeiss EM 10C electron microscope. Basal linear deposit (vesicular granular and collagenous material that lies external to the RPE basement membrane and is present within the collagenous layers of Bruch's membrane) and basal laminar deposit (vesicular granular and collagenous material that lies between the RPE plasma membrane and the RPE basement membrane) were recognised based on the descriptions of Green and Enger. ${ }^{18}$

\section{Results}

CASE 1

An 84 year old white man with a 1 month history of decreased vision in his right eye and visual acuity of 20/200 underwent surgical excision of a $750 \mu \mathrm{m}$ diameter subfoveal CNV (Fig 1A). Fluorescein angiography 2 weeks after surgery disclosed only mild focal hyperfluorescence and leakage at the perimeter of the previous location of the $\mathrm{CNV}$ in the late frames of the angiogram (Fig 1B, C). Seven weeks after surgery the visual acuity was $4 / 200$, and a well defined persistent CNV (900 $\mu \mathrm{m}$ 

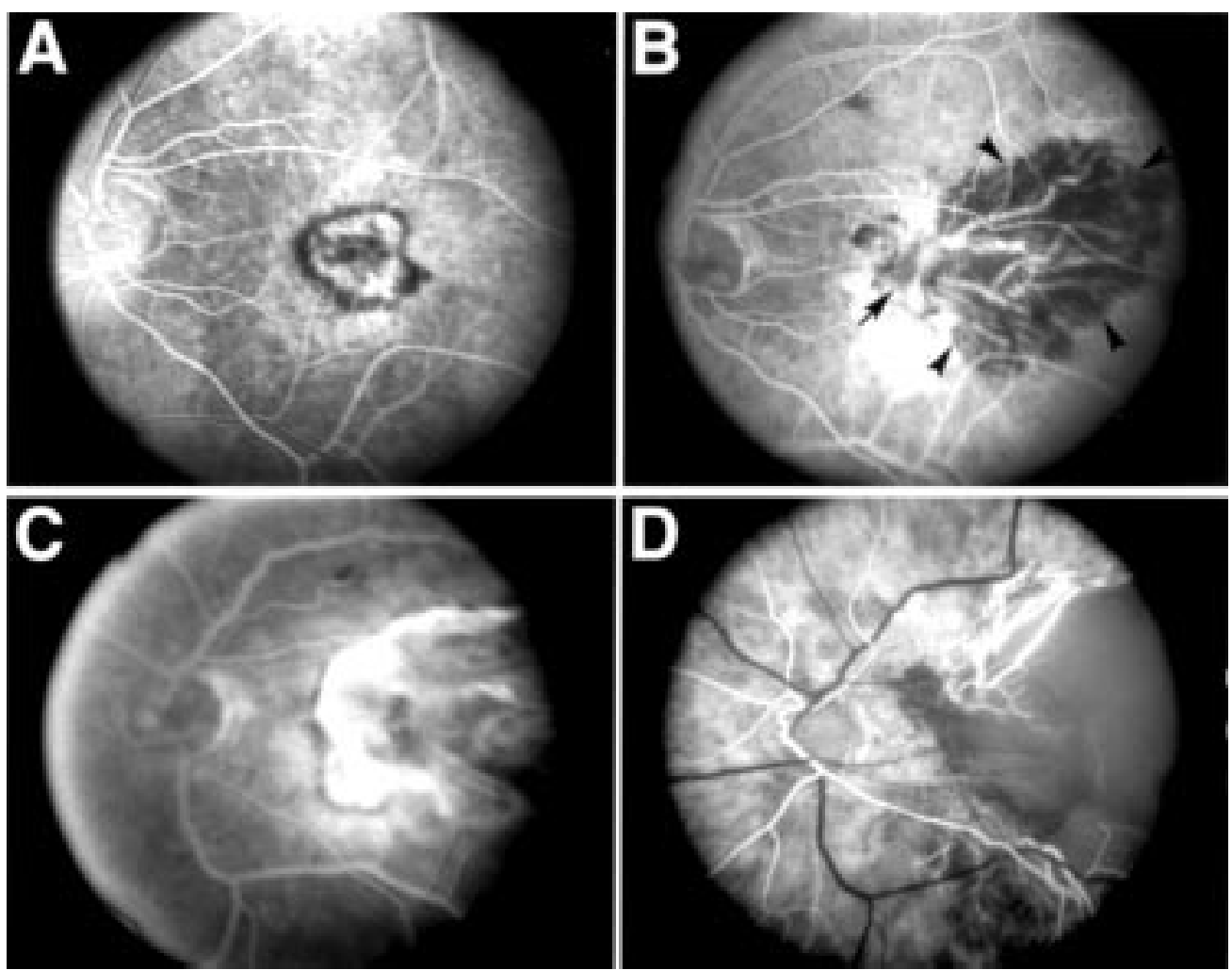

Figure 4 Case 2. (A) Preoperative fluorescein angiogram showing well demarcated subfoveal CNV with border of elevated blocked fluorescence. (B) Fluorescein angiogram taken 6 weeks after CNV excision shows choriocapillaris non-perfusion in the area of laser photocoagulation (arrowheads) and impaired choriocapillaris perfusion in the previous location of the CNV (arrow). (C) Fluorescein angiogram taken 7 months after surgery discloses a large, recurrent subfoveal CNV. (D) Fluorescein angiogram taken 6 months after excision of the recurrent CNV shows profound choroidal (including choriocapillaris) non-perfusion in the previous location of the CNV.

diameter) in the location of the previous CNV was identified clinically and on fluorescein angiography (Fig 1D). The patient underwent repeat CNV excision complicated by mild postoperative subretinal bleeding which resolved over 4 weeks. Three months after surgery, the vision was $3 / 200$, and examination disclosed RPE choriocapillaris atrophy in the location of the dissection and persistent choroidal neovascularisation at the perimeter of the dissection (Fig 1E).

Light microscopy of the primary excised surgical specimen disclosed collagen (Fig 2A) and vascular channels containing erythrocytes (Fig 2B). No RPE cells were noted, but some free melanin granules were identified. Ultrastructural study of the specimen disclosed fibrillar collagen and non-endothelial lined vascular channels containing extravasated erythrocytes (Fig 2B). Light and electron microscopy of the recurrent $\mathrm{CNV}$ showed that the external surface was lined by presumed RPE cells. Other parts of the neovascular complex contained presumed hyperplastic RPE cells in a collagenous matrix and extravasated erythrocytes (Fig 3A, B). Thin, perhaps newly formed, basement membrane like material lined the basal plasma membrane of presumed RPE (Fig 3B, inset).

CASE 2

A 63 year old man with a 3 month history of visual loss in his left eye and visual acuity of $20 / 200$ underwent surgical excision of a 1500 $\mu \mathrm{m}$ diameter subfoveal CNV (Fig 4A). Six weeks after surgery, the fluorescein angiogram disclosed choriocapillaris perfusion delay (Fig 4B). The best postoperative vision, 1 month later, was $20 / 400$. Seven months after surgery, a well defined recurrent CNV (2200 $\mu \mathrm{m}$ diameter) was diagnosed clinically and on fluorescein angiography (Fig 4C). Initially the patient declined further surgery, but 13 months after the first procedure, with visual acuity of $4 / 200$ and significant exudative macular detachment, he elected to undergo repeat $\mathrm{CNV}$ excision. Six months after surgery the visual acuity was $2 / 200$, and there was profound chorioretinal atrophy in the area previously occupied by the CNV. The fluorescein angiogram disclosed choriocapillaris non-perfusion in the previous location of the CNV (Fig 4D).

Light microscopy of the primary CNV showed collagen, fibrous tissue, and occasional presumed RPE cells containing melanin granules (Fig 5A). Ultrastructural examination showed that the external cleavage plane was lined by occasional presumed RPE cells with subjacent basal laminar deposit and discontinuous RPE basement membrane (Fig 5B). Fibrocytes, substantial amounts of fibrillar collagen, occasional macrophages, and endothelium lined vascular channels containing erythrocytes (anterior to the RPE) were also present. Light microscopy of the recurrent CNV disclosed fibrous tissue containing vascular channels, presumed RPE cells, and an external cleavage plane which varied and 

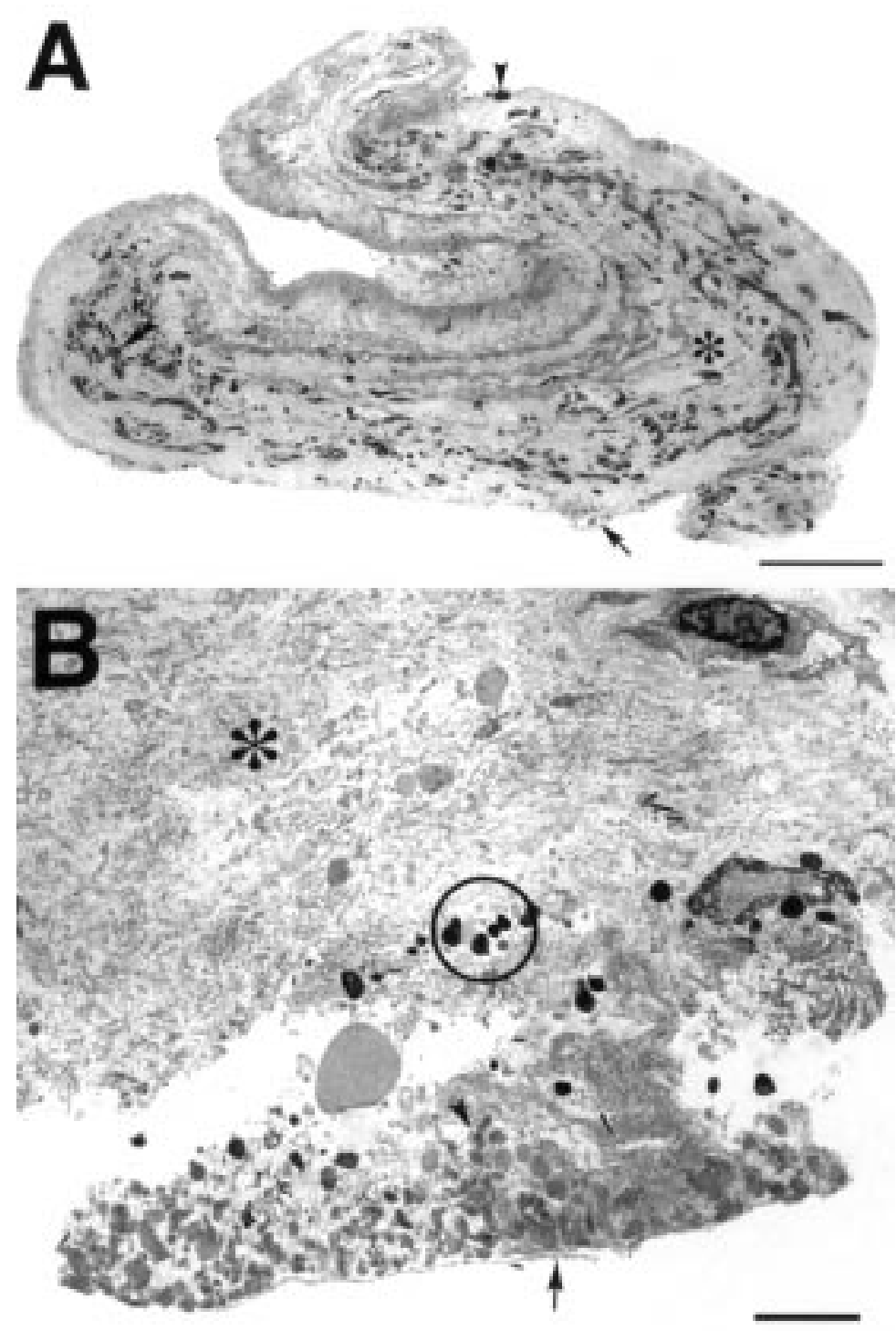

Figure 5 Case 2. (A) Photomicrograph of the primary CNV shows presumed RPE (arrowhead), collagen (asterisk), and blood (double arrowhead). Bar $=100 \mu \mathrm{m}$. Arrow shows location of $(B)$. (B) Transmission electron micrograph of $(A)$ shows that the specimen's external cleavage plane is lined by fragmented presumed RPE basement membrane (arrow) with overlying basal laminar deposit (arrowhead). Scattered melanin granules (circle) appear to be in cells or cell debris and probably represent the position of RPE that has been disrupted. Most of the specimen is composed of a loose matrix of collagen (asterisk). Bar $=5 \mu \mathrm{m}$.

included the RPE basement membrane and, in one portion of the specimen, Bruch's membrane (Fig 6A). Ultrastructural study of the recurrent CNV showed that the external cleavage plane varied and occurred at the following levels: external to basal laminar deposit; external to the RPE basement membrane; through the inner collagenous portion of Bruch's membrane; and possibly at the level of the choriocapillaris (Fig 6B). Multilayers of RPE cells with thickened multilaminar basement membrane enmeshed in a fibrillar collagen matrix were present in some portions of the neovascular complex (Fig 6B). Flattened cells containing melanin granules (presumed RPE) with flattened, oblique borders and thin basement membrane overlay fragments of inner Bruch's membrane (Fig 6B, C). These cells appeared to be migrating on Bruch's membrane in a plane between the inner collagenous lamina and the native RPE basement membrane. Portions of inner Bruch's membrane were also covered with multilayers of fibrocytes and fibrillar collagen, and acini of presumed RPE were observed (Fig 6C, D). In two areas, fragments of full thickness Bruch's membrane were enmeshed in a collagenous matrix containing fibrocytes. Endothelial lined vascular channels were present external to the Bruch's membrane fragment, and in some areas, abnormal choriocapillaris was present (Fig 6C). In some areas, the choriocapillaris was atrophic or absent between the pillars of Bruch's membrane suggesting preoperative choriocapillaris atrophy (Fig 6B, D, E).

\section{Discussion}

Recurrent/persistent choroidal neovascularisation following apparently successful CNV excision occurs in $0-40 \%$ of reported cases. ${ }^{8}{ }^{11-13} 16$ With longer follow up, the recurrence rate may be even higher. It is difficult to compare the recurrence rates among the reported retrospective series since the number of patients is small, the eligibility criteria sometimes differ, and the surgical technique varied. None of these studies prospectively compared foveal laser photocoagulation with submacular surgery.

In case 1 , we hypothesise that CNV persistence was the result of incomplete CNV excision. The presence of a neovascular complex with a diameter of $900 \mu \mathrm{m}$ in the same location as the primary CNV only 7 weeks after the first surgery supports the notion that the recurrence resulted from incomplete CNV removal. We cannot explain why the early postoperative angiograms did not show persistence of the CNV although the presence of subretinal fibrin, subretinal fibrous tissue, subretinal pigment, or a thin layer of sub-RPE blood might have blocked CNV fluorescence. Subretinal blood was not visible clinically and thus probably cannot account for these findings. It is also possible that some effect of the surgery itself may have temporarily altered the fluorescein leakage from the CNV. After the second surgery, choriocapillaris perfusion delay developed in the dissection bed in the area previously occupied by the CNV, as has been noted previously. ${ }^{1920}$ Choriocapillaris atrophy with overlying photoreceptor degeneration may partly underlie the limited visual outcome following subfoveal surgery for AMD.

Grossniklaus and coworkers found a positive correlation between the presence of Bruch's membrane components and the development of recurrent CNVs. ${ }^{14}$ Histopathology of the primary CNV in case 1 did not show Bruch's membrane components. There were relatively few vascular channels, however, which has been associated with incomplete removal of the vascular component of the $\mathrm{CNV}$ at surgery. ${ }^{14}$ The persistent CNV in case 1 exhibited numerous presumed RPE cells in a dense collagenous matrix. We believe that abnormal RPE with scarring of Bruch's membrane has repopulated the dissection bed. In laboratory experiments, Korte et $a l^{21-23}$ have shown that RPE death/absence results in the atrophy of subjacent choriocapillaris. Thus, abnormal 

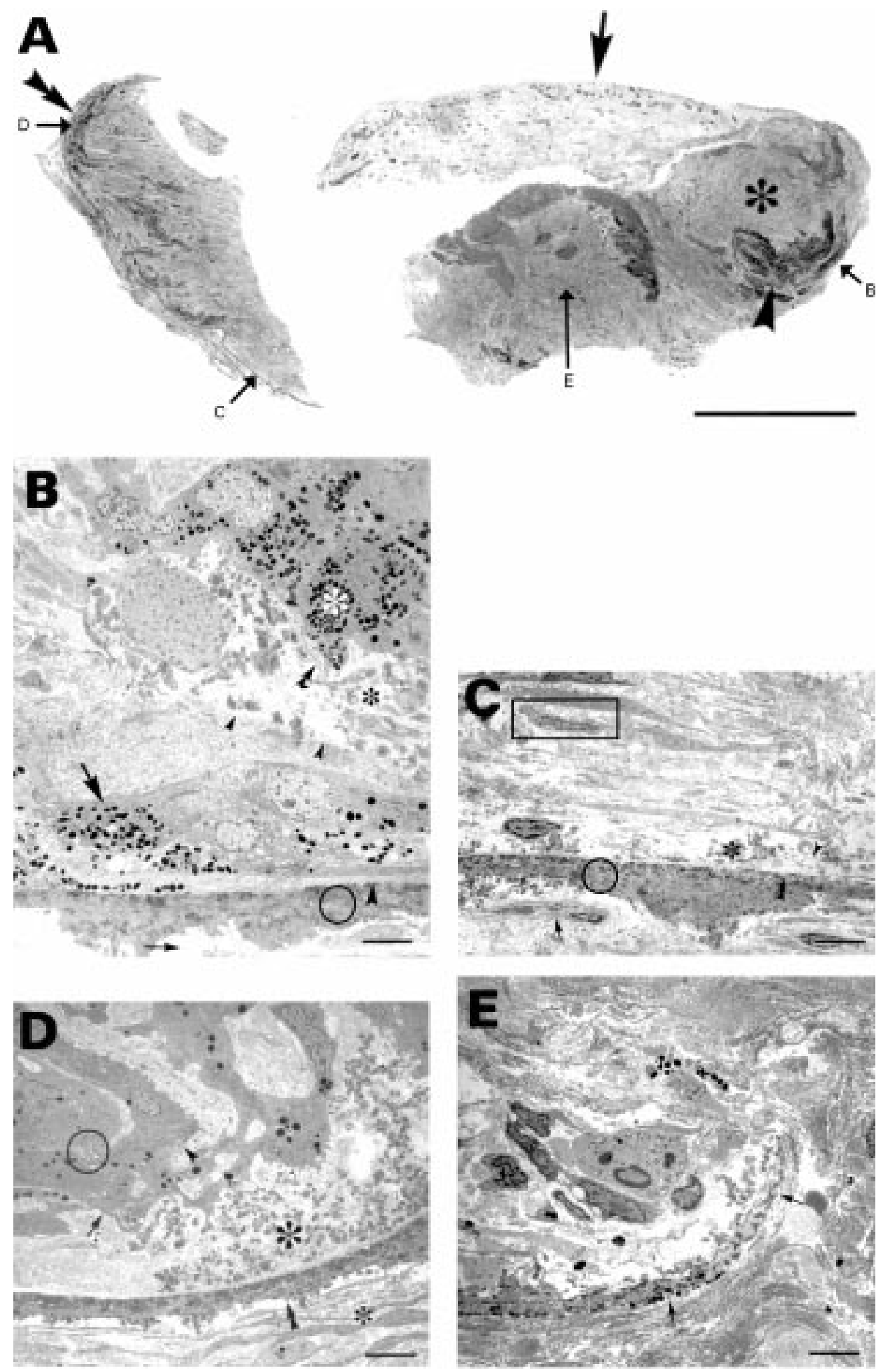

Figure 6 Case 2. (A) Photomicrograph of the recurrent CNV.In one area there is a loose collagenous network in which vessels and cellular elements are embedded (arrow). The other portion of the specimen contains more densely packed collagen (asterisk), presumed hyperplastic RPE (arrowhead), and large portions of Bruch's membrane (double arrowhead). Bar $=500$ um. The locations from which $(B),(C),(D)$, and $(E)$ are derived are indicated. $(B)$ Transmission electron micrograph of $(A)$ shows flattened pigment containing presumed RPE (arrow) apparently migrating on subjacent full thickness Bruch's membrane with basal linear deposit (circle) and underlying choriocapillaris atrophy (arrow). Presumed RPE basement membrane (arrowheads) is present in several locations. Presumed hyperplastic RPE (white asterisk) with reduplicated basement membrane (double arrowheads) overlie basal laminar deposit (black asterisk). Bar $=5 \mu \mathrm{m} .(C)$ Transmission electron micrograph of $(A)$ shows Bruch's membrane thickened by basal linear deposit (circle). An abnormal choriocapillaris endothelial cell is present between the pillars of Bruch's membrane (small arrow). Basal laminar deposit (asterisk), RPE basement membrane (arrowhead), and fibrocytes (rectangle) in a collagenous matrix overlie Bruch's membrane. Bar $=5 \mu \mathrm{m}$. (D) Transmission electron micrograph of $(A)$ showing a fragment of Bruch's membrane (double arrowhead) enmeshed in collagen (small asterisk) with overlying basal laminar deposit (large asterisk) and presumed hyperplastic RPE (arrow). The presumed RPE exhibits basement membrane (arrowhead) and acinus formation (circle). Bar = 5 um. (E) Transmission electron micrograph of $(A)$ showing a curled edge of a fragment of Bruch's membrane (arrows) embedded in collagen. Bar $=5 \mu \mathrm{m}$. 
RPE resurfacing of Bruch's membrane may underlie the choriocapillaris atrophy seen angiographically. Bruch's membrane scarring, abnormal RPE resurfacing of the dissection bed, and subsequent choriocapillaris atrophy may underlie the poor final visual acuity.

Case 2 demonstrated a significant delay between excision of the primary neovascular complex and $\mathrm{CNV}$ recurrence. In this case, the recurrence grew from the edge of the area occupied previously by the CNV. This patient demonstrated choriocapillaris non-perfusion after both the first and second operations but in a new location the second time. While the first specimen showed occasional presumed $\mathrm{RPE}$, discontinuous RPE basement membrane, and occasional fragments of inner Bruch's membrane with basal laminar deposit at one edge, the second specimen disclosed an external cleavage plane involving RPE basement membrane and fragments of Bruch's membrane in a matrix of fibrillar collagen and fibrocytes. This suggests that CNV removal can be followed by scarring of Bruch's membrane and an abnormal arrangement of RPE. Some of the choroidal atrophy and scarring may have been due to laser photocoagulation during the first surgery. Macrophages, presumed metaplastic RPE, and fibroblasts were present, suggesting that repopulation of the dissection bed involves not only RPE migration, presumably from the perimeter of the dissection, but also the migration of inflammatory cells to the area. The fibroblastlike cells could have arisen from RPE metaplasia or from blood derived cells. We suspect that the recurrence in this case was due to incomplete CNV excision.

Studies have shown that CNV may be present but not be evident clinically or on fluorescein angiography, ${ }^{24}{ }^{25}$ which makes it difficult to distinguish persistent from recurrent CNVs except on arbitrary grounds-that is, recurrent CNVs are those detected clinically 6 weeks or more after treatment. Green and Enger found an average of over two choroidal feeding vessels for each $\mathrm{CNV}$, which supports the notion that many recurrences are due to incomplete excision. ${ }^{18} 25$ The angiographic heterogeneity of CNVs often makes it difficult recognise their edges. Thus, without adequate follow up, it may be difficult to be certain that the entire CNV has been removed simply by careful scrutiny of the pre- and postoperative fluorescein angiograms or by comparison of the excised tissue with the preoperative fluorescein angiogram.

Based on the clinical and histological data of these two cases, we think that several variables may affect the development of persistent and/or recurrent CNVs. The anatomical location of the neovascular complex may be quite important. Whether the CNV lies underneath or above the RPE or within Bruch's membrane may affect whether the excision is complete or not and may influence the amount of surgical trauma to Bruch's membrane associated with $\mathrm{CNV}$ removal. In AMD, CNVs usually lie within abnormally thickened Bruch's membrane and underlie the RPE. CNVs associated with the presumed ocular histoplasmosis syndrome may grow anterior to the RPE into the subneurosensory retinal space, or they may have a pattern of more diffuse growth and involvement on both sides of the RPE. ${ }^{26}$ Our clinical impression was that the primary CNV of case 1 lay anterior to the RPE which may account for the incomplete excision (as a subRPE component probably existed at the time of surgery). In case 2, the CNV was posterior to the RPE. Grossniklaus and coworkers demonstrated that half of the CNVs that recurred lacked vascular channels in the primary membrane, raising the possibility that in case 2 , the vascular component of the $\mathrm{CNV}$ was removed incompletely at surgery. ${ }^{14}$

Abnormal RPE repopulation of the dissection bed and lack of restoration of the outer blood-retinal barrier might play an important role in CNV recurrence. Most of the presumed RPE cells that we found in the recurrent CNV specimens were abnormal-for example, nonpigmented, hyperplastic, and metaplastic. Several factors may account for this including the age of the patients and an abnormal microenvironment. In vitro experiments demonstrate a high rate of metaplastic change and a low mitotic index in cultured RPE from people over the age of $75 .^{27}$ The presence of basal laminar deposit, basal linear deposit, the lack of RPE basement membrane, and/or the exposure of the abnormal inner part of Bruch's membrane might affect the ingrowth of the $\mathrm{RPE}$ into the dissection bed. Furthermore, mechanical trauma during surgery and the leakage of inflammatory mediators from the retina and/or choriocapillaris into the subretinal space might play an important role in the abnormal repopulation of the dissection bed. ${ }^{28}$ Because of the anatomical location of the CNV (sub-RPE-intra-Bruch's membrane), the removal of the neovascular complex might damage Bruch's membrane further. Thus, CNV excision might be associated with new breaks in Bruch's membrane allowing new vessels to grow through. ${ }^{2425} 29$

Bruch's membrane scarring and inflammatory cells in the excised secondary neovascular complexes indicate the presence of an inflammatory reaction. Several investigators have suggested inflammation as a possible cause of CNVs in AMD. ${ }^{24}{ }^{29-32}$ Necropsy findings are consistent with the concept that CNVs in AMD are localised intra-Bruch's membrane granulation tissue associated with diffuse drusen. Histological studies of surgically excised CNVs confirm the presence of inflammatory cells including foreign body giant cells. ${ }^{14} 15$ Immunohistochemistry performed on surgically excised CNVs has shown the presence of RPE, inflammatory cells, fibrocytes, and myofibroblasts and also the presence of collagen types I, III, IV, and V, fibronectin, and laminin in the extracellular matrix of the CNV..$^{15}$ Surgical trauma, especially the removal of $\mathrm{RPE}$, could promote the leakage of inflammatory mediators from the choriocapillaris or retina that might start or aggravate this inflammatory reaction. Heriot and Machemer demonstrated in rabbits that within a day of 
RPE debridement, there was choriocapillaris endothelial enlargement and infiltration with polymorphonuclear leucocytes and spindleshaped cells that resembled fibrocytes. ${ }^{33}$ These inflammatory changes may, through cell mediated tissue contraction, result in adjacent RPE tears or new breaks in Bruch's membrane with possible development of new areas of neovascularisation. ${ }^{15}$ Thus, surgery itself might be a stimulus which starts a non-specific wound repair-like response with higher risk for the development of new vessels and Bruch's membrane scarring.

In summary, we suspect most cases of recurrent CNVs are the result of incomplete excision of clinically and angiographically occult components of the CNV, the growth of which may result from various factors including postoperative inflammation, abnormal Bruch's membrane resurfacing by native RPE at the perimeter of the dissection, and scarring (that is, granulation tissue growth) of Bruch's membrane. These changes may also underlie the lack of significant visual improvement often seen after $\mathrm{CNV}$ excision in AMD.

Supported in part by That Man May See, Inc, The Foundation Fighting Blindness, and Research to Prevent Blindness, Inc (MAZ).

1 Bressler SB, Bressler NM, Fine SL, et al. Natural course of choroidal neovascular membranes within the foveal avascular zone in senile macular degeneration. Am $\mathcal{F}$ Ophthalmol 1982;93:157-63

2 Coscas G, Subrane G, Ramahefasolo C, et al. Perifoveal laser treatment for subfoveal choroidal new vessels in agerelated macular degeneration. Results of randomized clinical trial. Arch Ophthalmol 1991;109:1258-65.

3 Macular Photocoagulation Study Group. Laser photocoagulation of subfoveal neovascular lesion in age-related macular degeneration. Results of a randomized clinical trial. Arch Ophthalmol 1991;109:1232-41.

4 Macular Photocoagulation Study Group. Laser photocoagulation of subfoveal neovascular lesion in age-related macular degeneration. Updated findings from two clinical macular degeneration. Updated findings

5 Macular Photocoagulation Study Group. Subfoveal neovasMacular Photocoagulation Study Group. Subfoveal neovascular lesions in age-related macular degeneration. Guidelines for evaluation and treatment in the Macular Photo

6 Freund KB, Yannuzzi LA, Sorenson JA. Age-related macular degeneration and choroidal neovascularization. $A m \mathcal{F}$ Ophthalmol 1993;115:786-91.

7 D'Amato RJ, Loughnan MS, Flynn E, et al. Thalidomide is an inhibitor of angiogenesis. Proc Natl Acad Sci USA 1994 91:4082-5.

8 Berger AS, Kaplan HJ. Clinical experience with the surgical removal of subfoveal neovascular membranes. Ophthalmology 1992;99:969-75.

9 Chakravarthy U, Houston RF, Archer DB. Treatment of age related subfoveal neovascular membranes by teletherapy: a pilot study. Br f Ophthalmol 1993;77:265-73.

10 Fung WE. Interferon alpha 2 a for treatment of age related macular degeneration. Am f Ophthalmol 1991;112:349-50.
11 Lambert HM, Capone A Jr, Aaberg TM, et al. Surgical exciion of sion of subfoveal neovascular membranes in age-related
macular degeneration. Am $\mathcal{f}$ Ophthalmol 1992;113:257-62.

12 Thomas MA, Dickenson J Ophthalmol 1992;113:257-62. Thomas MA, Dickenson JD, Melberg MD, et al. Visual
results after surgical removal of subfoveal choroidal neovascular membranes. Ophthalmology 1994;101:138496.

13 Ormerod LD, Puklin JE, Frank RN. Long-term outcomes after the surgical removal of advanced subfoveal neovascular membranes in age related macular degeneration. lar membranes in age related mac

14 Grossniklaus HE, Hutchinson AK, Capone A Jr, et al. Clinicopathologic features of surgically excised choroidal neovascular membranes. Ophthalmology 1994;101:1099111.

15 Lopez PF, Grossniklaus HE, Lambert HM, et al. Pathologic features of surgically excised subretinal neovascular membranes in age-related macular degeneration. Am f Ophthalmol 1991;112:647-56.

16 Melberg N, Thomas M. Successful feeder vessel laser treatment of recurrent neovascularization following subfoveal surgery. Arch Ophthalmol 1996;114:224-6.

17 Nasir M, Sugino I, Zarbin MA. Clinicopathologic correlation of excised primary and recurrent choroidal neovascuarization (rCNV) in age-related macular degeneration. (ARVO abstracts) Invest Ophthalmol Vis Sci (Suppl) 1994;35: 1504 .

18 Green WR, Enger C. Age-related macular degeneration histopathologic studies. Ophthalmology 1993;100:1519-35.

19 Desai VN, Del Priore LV, Kaplan HJ. Choriocapillaris atrophy after submacular surgery in presumed ocular histoplasmosis syndrome. Arch Ophthalmol 1995;113:408-9.

20 Nasir M, Sugino IK, Zarbin MA. Choriocapillaris atrophy as a complication of surgical excision of choroidal neovascular membranes. Br $\mathcal{F}$ Ophthalmol 1997;81:481-9.

21 Korte GE, Reppucci V, Henkind P. RPE destruction causes choriocapillary atrophy. Invest Ophthalmol Vis Sci 1984;25: 1135-45.

22 Korte GE, Gerszberg T, Pua F, et al. Choriocapillaris atrophy after experimental destruction of the retinal pigment epithelium in the rat. Acta Anat 1986;127:171-5.

23 Korte G, Burns M, Bellhorn R. Epithelium-capillary interactions in the eye: the retinal pigment epithelium and the choriocapillaris. Int Rev Cytol 1989;1142:221-48.

24 Green WR, Key SN. Senile macular degeneration. A histopathologic study. Trans Am Ophthalmol Soc 1977;75: $180-254$.

25 Green WR. Clinicopathologic studies of treated choroidal neovascular membranes. A review and report of two cases. Retina 1991;11:328-56.

26 Gass JD. Biomicroscopic and histopathologic considerations regarding feasibility of surgical excision of subfoveal neovascular membranes. Am $\mathcal{f}$ Ophthalmol 1994;118:28598.

27 Flood MT, Gouras P, Kjelbye H. Growth characteristics and ultrastructure of human retinal pigment epithelium in vitro. Invest Ophthalmol Vis Sci 1980;19:1309-20.

28 Reddy VM, Zamora RL, Kaplan HJ. Distribution of growth factors in subfoveal neovascular membranes in age related macular degeneration and presumed ocular histoplasmosis syndrome. Am f Ophthalmol 1995;120:291-301.

29 Sarks SH. New vessel formation beneath the retinal pigment epithelium in senile eyes. Brf Ophthalmol 1973;57:951-65.

30 Loffler KU, Lee WR. Basal linear deposit in the human macula. Graefes Arch Clin Exp Ophthalmol 1986;224:493501.

31 Penfold PL, Killingsworth MC, Sarks SH. Senile macular degeneration: the involvement of immunocompetent cells. Graefes Arch Clin Exp Ophthalmol 1985;225:69-76.

32 Penfold PL, Provis JM, Billson FA. Age-related macular degeneration: ultrastructural studies of the relationship of leucocytes to angiogenesis. Graefes Arch Clin Exp Ophthalmol 1987;225:70-6.

33 Heriot WJ, Machemer R. Pigment epithelial repair. Graefes Arch Clin Exp Ophthal 1992;230:91-100. 